\title{
Endocarditis, Meningitis and Pneumocystis Pneumonia
}

\author{
Filipa Silva ${ }^{1}$, Sérgio Andrade ${ }^{2}$, João Vilaça ${ }^{2}$, Cristiana Paulo², Filipe Conceição ${ }^{2}$ \\ ${ }^{1}$ Internal Medicine Department, Centro Hospitalar de São João, Porto, Portugal \\ ${ }^{2}$ Intermediate Care Unit of the Emergency Department, Centro Hospitalar de São João, Porto, Portugal
}

Received: 15/11/2016

Accepted: 04/12/2016

Published: 29/12/2016

How to cite this article: Silva_F, AndradeS, Vilaça J, PauloC, ConceiçãoF. Endocarditis, meningitis and pneumocystis pneumonia. EJCRIM 2016;3: doi:10.12890/2016_000535

Conflicts of Interests: The Authors declare that there are no competing interests.

This article is licensed under a Commons Attribution Non-Commercial 4.0 License

\section{ABSTRACT}

Infective endocarditis (IE) is associated with high morbidity and mortality despite advances in antibiotic and surgical treatment. Systemic embolism occurs in up to $49 \%$ of IE patients and may involve the major arteries, limb arteries, viscera and the central nervous system. In this report we describe a 60-year-old female patient with a history of acute lymphoblastic leukaemia who presented with endocarditis manifesting as stroke, acute limb ischaemia and meningitis. Early diagnosis is essential since treatment lowers the risk of embolism, with most events occurring within 2 weeks of treatment initiation.

\section{LEARNING POINTS}

- Infective endocarditis has a very variable clinical presentation and should be suspected in patients with multisystemic involvement especially if vascular and neurological systems are affected.

- As early diagnosis is essential since treatment lowers the risk of embolism, echocardiography should be more widely available and possibly become a core medical skill.

- Because of the high mortality, a high index of suspicion and low threshold for investigation is essential in at-risk groups.

\section{KEYWORDS}

Infective endocarditis; complications; embolic stroke; acute limb ischemia; meningitis

\section{INTRODUCTION}

Infective endocarditis (IE) is associated with a broad array of complications, with embolism being the most feared ${ }^{[1]}$. Despite improved diagnostic and therapeutic strategies, IE is still associated with high in-hospital mortality and a high incidence of embolic events. The wide range of complications emphasizes the heterogeneity of the disease and the need for baseline risk stratification in order to identify high-risk patients and implement more aggressive treatment ${ }^{[2]}$.

The clinical manifestations of IE are highly variable, ranging from low-grade fever and non-specific symptoms such as chills and anorexia to embolic complications. Duke's criteria are the most common criteria used to diagnose $\mathrm{E}^{[3]}$.

Neurological manifestations are the most frequent extra-cardiac complications of IE, and are dominated by stroke. Atrial fibrillation and IE are two of the most common causes of embolic stroke ${ }^{[4]}$ which, as in our case, might be the first sign of IE.

\section{CASE DESCRIPTION}

We present the case of a 60-year-old Caucasian woman with a history of acute lymphoblastic leukaemia diagnosed in February, 2016 which was refractory to first line treatment but achieved complete remission with the protocol of fludarabine, cytarabine, idarubicin and granulocyte colony-stimulating factor. In July, the patient was diagnosed with acute fibrinous and organising pneumonia and was started on steroids. 
One week prior to admission the patient began to complain of fever and paraesthesia in the right limb. She was evaluated in the emergency department (ED) and the work-up revealed anaemia of $8.6 \mathrm{~g} / \mathrm{dl}$, thrombocytopenia $\left(70,000 / \mathrm{mm}^{3}\right)$, leukocytosis $\left(14,000 \times 10^{9} / \mathrm{I}\right)$ and C-reactive protein of $221 \mathrm{mg} / \mathrm{l}$ (normal <3 mg/l), acute renal failure (creatinine $1.3 \mathrm{mg} / \mathrm{dl}$ ) and high LDH (1,947 U/I). Serum electrolytes, and renal and liver function tests were within the normal range; urine analysis, chest $\mathrm{x}$-ray and abdominal ultrasound were unremarkable. The patient was observed by a vascular surgeon who described normal pulse palpation and Doppler in the affected limb, and was discharged medicated with amoxicillin and clavulanic acid. After 4 days the fever still persisted and the patient became confused and disoriented and was admitted again to the ED.

On initial evaluation, her temperature was $39^{\circ} \mathrm{C}$, blood pressure was $129 / 78 \mathrm{mmHg}$, pulse was 105 beats/min and oxygen saturation was $96 \%$ in room air. She was conscious, disoriented and had paraesthesia of the right lower limb (that resolved), without any other deficits or signs of meningeal irritation. A brain CT scan excluded ischaemic or haemorrhagic lesions. Work-up showed haemoglobin of $8.6 \mathrm{~g} / \mathrm{dl}$, thrombocytopenia $\left(52,000 / \mathrm{mm}^{3}\right)$, leukocytes of $9,680 \times 10^{9} / \mathrm{l}$ and C-reactive protein of $199 \mathrm{mg} / \mathrm{l}$, indirect hyperbilirubinaemia (1.53 mg/dl) and haptoglobin $<8 \mathrm{mg} / \mathrm{dl}$. The Coombs test was negative, and renal function, electrolytes and coagulation profile were within normal limits. Peripheral blood smear revealed rare schistocytes. Chest $x$-ray was normal.

On admission a lumbar puncture (LP) was performed and revealed 1,126 cells with a predominance of polymorphonuclear cells (89.7\%), normal glucose $(64 \mathrm{~g} / \mathrm{l})$ and a low protein concentration $(0.09 \mathrm{~g} / \mathrm{l})$. The patient was started empirically on meropenem, vancomycin and ampicillin. CSF polymerase chain reaction (PCR) for HSV 1 and 2, human herpesvirus 6, enterovirus and listeria came back negative. The China ink test and CSF bacterial, mycobacterial and mycologic cultures were negative.

On the second day, the patient developed prostration, dysarthria and right hemiparesis that recovered completely in about 1 hour. A brain CT scan excluded ischaemic or haemorrhagic lesions. By the end of the day she had dyspnoea with acute respiratory insufficiency. A chest $\mathrm{x}$-ray revealed bilateral pulmonary infiltrates and a CT scan showed consolidation of the right upper lobe and diffuse ground-glass opacity in both lung fields with bilateral pleural effusion. Since she was an immunocompromised patient, pneumocystis pneumonia was considered and she was treated with trimethoprim-sulfamethoxazole combined with corticosteroids. Bronchofibroscopy with alveolar lavage was positive and PCR showed Pneumocystis jiroveci.

The next day the right lower limb was pale and pulseless suggesting acute limb ischaemia and the patient underwent an emergency thromboembolectomy with good results. The LP was repeated and showed improvement with 147 cells ( $92.5 \%$ polymorphonuclear) and glucose $56 \mathrm{mg} / \mathrm{dl}$ with a normal protein level. Immunophenotyping of LCR excluded CNS involvement due to leukaemia. An autoimmune study and serological tests for HIV, hepatitis, syphilis, CMV, EBV, herpes simplex 1 and 2, toxoplasmosis, mycoplasma pneumonia and varicella were also negative.

On the fifth day, the patient experienced dysarthria, right hemiparesis and blindness in the left eye. A brain CT scan showed hypodensity in the left-sided talamo-capsular area compatible with ischaemic stroke.

Due to these recurring embolic events, endocarditis was considered and transthoracic (TTE) and then transoesophageal echocardiography (TEE) was performed. It revealed a large multilobulated vegetation $(18 \times 12 \mathrm{~mm})$ adhering to the free border of the anterior leaflet of the mitral valve causing severe mitral regurgitation (Fig. 1). The serum galactomannan antigen was positive and, despite the possibility of a false positive, voriconazol was started because of the patient's clinical severity.

The patient was referred for cardiothoracic surgery but intervention was declined due to poor functional status. Her clinical condition deteriorated during the following days and she had another embolic stroke. She died on the seventh day.

\section{DISCUSSION}

With this clinical case the authors seek to draw attention to one of the most devastating consequences of endocarditis, that is systemic embolization, and also the rare association with meningitis, both of which have high rates of poor outcome. A prompt diagnosis is essential since the risk of embolism is lower after initiation of treatment, with most events occurring during the first 2 weeks of treatment ${ }^{[3]}$.

Neurological complications develop in $20-40 \%$ of patients, the most frequent being intracerebral haemorrhage, cerebral infarction, mycotic aneurysms and purulent or aseptic meningitis. Ischaemic strokes most commonly affect the territory of the middle cerebral artery, often involve multiple vessels and are prone to haemorrhagic transformation ${ }^{[2]}$.

A higher risk of embolism has also been found with staphylococcal and fungal infections, a vegetation size of $>10 \mathrm{~mm}$, high mobility, associated embolic events and mitral valve (or multivalvular) involvement ${ }^{[5]}$.

As happened in our patient, the discovery of such neurological complications demands careful consideration since it may alter decisionmaking regarding surgical management of the underlying disease. In fact, there is a significant risk of postoperative neurological deterioration or death in patients with a recent central nervous system complication of IE, and so such an event is considered a relative contraindication for 

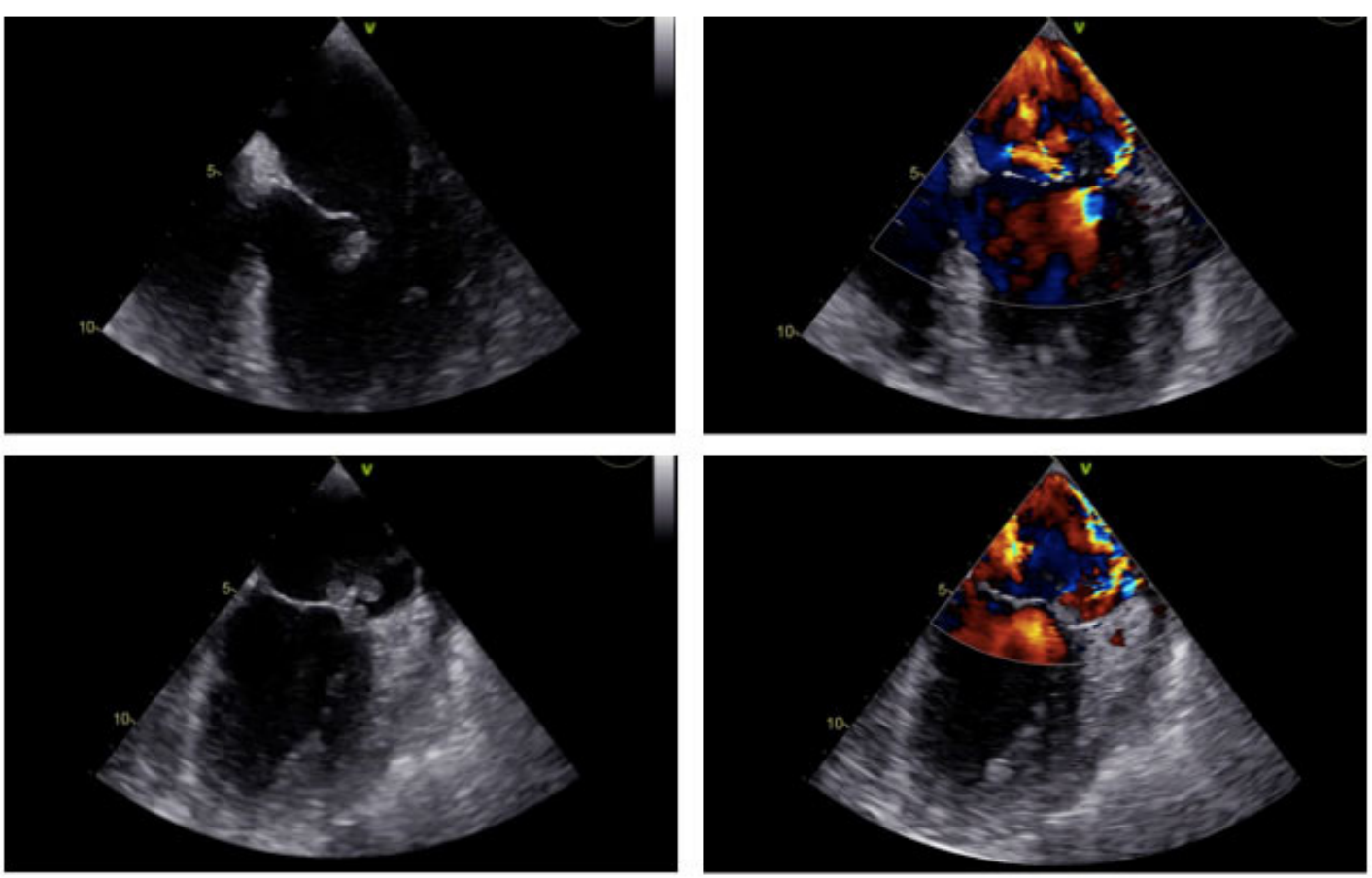

Figure 1. Transoesophageal echocardiogram showing a large multilobulated vegetation adhering to the free border of the anterior leaflet of the mitral valve resulting in severe mitral regurgitation

early cardiac surgery. Since neurological complications of IE are frequent and increase mortality to about 60\%, the appropriate management of these patients needs to be identified. Unfortunately, the literature does not currently provide suitable information ${ }^{[2]}$.

Acute limb ischemia (ALI) is a common vascular emergency. The incidence based on reported case series varies and ranges from 14 in 285 (4.9\%) to 7 in 47 (7.7\%). The best treatment for this complication of IE has not been determined. For many years, surgical intervention was performed and entailed significant morbidity and mortality. Thrombolysis is avoided because of the risk of haemorrhage, and percutaneous mechanical thrombectomy (PMT) may be the best option ${ }^{[6]}$.

Another interesting aspect of this case is the association with meningitis. In fact, bacterial meningitis is often related to other foci of infection outside the central nervous system such as pneumonia, sinusitis or otitis. An uncommon focus of bacterial meningitis is IE, identified in only $2 \%$ of patients, which may occur during bacterial endocarditis caused by pyogenic organisms such as Staphylococcus aureus and enterococci. In more chronic bacterial endocarditis (formerly known as subacute bacterial endocarditis), sterile emboli to the brain may cause signs of meningeal irritation and cerebrospinal fluid pleocytosis. The reverse may also occur ${ }^{[2]}$.

This case highlights two major complications of endocarditis: systemic embolization (stroke and acute limb ischaemia) and rare involvement of the central nervous system (aseptic meningitis). Both of these conditions have high rates of unfavourable outcome, which reinforce the need to maintain a high level of suspicion to allow prompt diagnosis and treatment.

\section{REFERENCES}

1. Habib G. Embolic risk in subacute bacterial endocarditis: role of transesophageal echocardiography. Curr Cardiol Rep 2003;5:129-136.

2. Morris N, Marcelo M, Lyons J, Samuels M. Neurologic complications in infective endocarditis: identification, management and impact on cardiac surgery. Neurohospitalist 2014;4:213-222.

3. Salehian O, Chan K. Systemic embolism in endocarditis: incidence, risk factors, clinical significance and treatment strategies. Endocarditis: diagnosis and management. 2th ed. Springer; 2016.

4. Tahir H, Raza M, Daruwalla V, Khakwani Z. Acute embolic ischemic stroke as the sole presentation of subacute enterococcal endocarditis. Am J Med Case Rep 2016;4:212-215.

5. Vilacosta I, Graupner C, San Román J, Sarriá C, Ronderos R, Fernández C. Risk of embolization after institution of antibiotic therapy for infective endocarditis. J Am Coll Cardiol 2002;39:1489-1495.

6. Rajeev M, George J. Endovascular management of acute limb ischemia from septic emboli. Vasc Dis Manage 2013;10:E71-E74. 